\title{
DEFLECTION OF COSMIC RAYS BY A MAGNETIC FIELD
}

\author{
By L. F. Curtiss
}

\section{ABSTRACT}

A continuation of earlier experiments on this subject (Phys. Rev., vol. 34, p. 1931, 1929; vol. 35, p, 1433, 1930), gives decisive evidence that a considerable fraction of an unfiltered cosmic-ray beam can be deviated by a magnetic field of 7,000 gauss. Using four Geiger-Müller tube counters, coincidences were observed with and without a magnetic field. The arrangement was such that electrons in the beam, possessing energies corresponding to $10^{9}$ volts or less, could be prevented from producing coincidences. Observations were made for a total of 615 hours without the field and 508 hours with the field, in alternating periods of about 48 hours. The average number of coincidences without the magnetic field was found to be $1.31 \pm 0.09$ per hour and with the magnetic field $0.88 \pm 0.14$ per hour. This indicates that, for approximately 70 per cent of the cosmic-ray particles, the deviation is less than that for $10^{9}$ volt electrons. This harder portion of the beam may contain, in addition to electrons of higher energy than $10^{9}$ electron volts, protons of $5 \times 10^{8}$ electron volts and neutrons. These data indicate that the results obtained by Rossi (Nature, vol. 128, p. $300 ; 1931$ ) and of Motth-Smith (Phys. Rev., vol. 37, p. 1001; 1931), showing no effect of the magnetic field, were affected by scattering or absorption in the iron core in which the magnetic field was produced.

\section{CONTENTS}

I. Introduction

II. Experimental arrangement. 816

III. Observations

IV. Discussion

V. Acknowledgments

\section{INTRODUCTION}

In a letter ${ }^{1}$ to the Physical Review for November, 1929, a report was made of experiments with Geiger-Müller tube counters which seemed to show that cosmic rays consisted, in part at least, of electrically charged particles which could be deflected by a magnetic field. These observations were made with a pair of tube counters, the coincidences between which were observed by making simultaneous records on a paper tape with dotting pens actuated by the counters through amplifiers. Although qualitatively there was indication of a decrease on applying a magnetic field between the counters, the method was too crude to serve as a basis for a definite decision of the point in question.

The experiments were, therefore, repeated, using the vacuum tube circuit developed by Bothe ${ }^{2}$ for resolving coincidences electrically and following the suggestion by Tuve ${ }^{3}$ of using more than two counters so that a more sharply defined beam of cosmic rays may be studied.

\footnotetext{
1 Phys. Rev., vol. 34, p. 1391, 1929.

2 W. Bothe, ZS. f. Phys., vol. 59, p. 1, 1929.

${ }_{3}$ M. Tuve, Phys. Rev., vol. 35, p. 651, 1930.
} 
These experiments were made with three counters, the results of which were reported to the Physical Society ${ }^{4}$ in April, 1930. These data also pointed to a magnetic deviation of a part of the cosmic-ray beam. A decrease of the order of 25 per cent in coincidence was observed on applying a magnetic field of 7,000 gauss to the path of the rays between two of the counters. These observations were made with considerable difficulty. The counters were necessarily of a small size, since they could be no longer than the air gap of the electromagnet $(5 \mathrm{~cm})$. This reduced the sensitivity of the arrangement, requiring, therefore, long periods of observation. Much trouble was also encountered with the Bothe screen grid vacuum tube circuit as modified for use with three counters. An even more serious source of trouble and possible error was discovered when it was noticed that the counters themselves are very considerably affected by changes of temperature. Sealed counters, described elsewhere, ${ }^{5}$ were used for these experiments. The rate of counting at a given voltage falls off rapidly as the temperature is increased. Some counters have been found to cease counting entirely when their temperature is raised $10^{\circ}$ or $15^{\circ}$. This effect has not been investigated, but it is presumably caused by the increase of vapor pressure of nonpermanent gases and more volatile substances present in the partially exhausted counters. It could easily falsify observations made with the magnetic field, since the temperature of the windings of the electromagnet rises considerably during the prolonged periods of observations, causing the temperature of some of the counters to rise also.

Another disturbing condition in the former experiments was the presence in the building of several grams of radium which, although stored at some distance in a safe with lead walls 6 inches thick, nevertheless increased the rate of counting. This penetrating radiation could not be screened off readily and was responsible for a large number of accidental coincidences, these being proportional to the rate of counting of each counter.

Interest in the question of the magnetic deviation of cosmic rays was increased by the reports made by Rossi ${ }^{6}$ and by Mott-Smith ${ }^{7}$ that no deflection was observed when cosmic ray beams pass through magnetized iron. It is of importance to make certain of the earlier results. Therefore the investigation has been continued under improved conditions designed to eliminate as far as possible difficulties and sources of error encountered in the earlier experiments. This naper gives an account of the results which have been obtained.

\section{EXPERIMENTAL ARRANGEMENT}

The most annoying difficulties encountered in the previous experiments arose from the presence of a relatively large number of accidental coincidences and from changes in counting rate due to temperature changes. Particular attention was given to the elimination of these disturbances.

Considerable reduction in the number of accidental coincidences was effected by the removal of the apparatus from the building in which

\footnotetext{
4 Phys. Rev., vol. 35, p. 1433, 1930.

3 B. S. Jour. Research, vol. 4 (RP 165), p. 593, 1930.

B B. Rossi, Nature, vol. 128 , p. $300,1931$.

? L M. Mott-Smith, Phys. Rev., vol. 37, p. 1001, 1931.
} 
radium is stored. Since the accidentals are proportional to the product of the rate of the individual counters, the importance of this change, which reduces simultaneously the rate of all counters, is obvious. It seemed desirable, however, to reduce these accidentals even further, in.view of the long periods covered by the observations. At most, only a few genuine coincidences per hour could be expected so that it was desirable to reduce as far as possible the probability of any accidental coincidences.

The most effective method of reducing accidentals is to increase the number of counters. Accordingly a fourth counter, added to the previous arrangement, had the desired effect of reducing the accidental coincidences practically to zero. The advanatge of additional counters can best be shown by computing the accidentals for the case of two and four counters under otherwise similar conditions. For two counters the expected number, $E$, of accidentals is given by

$$
E=2 N_{1} N_{2} \tau
$$

where $N_{1}$ is the average rate of counting of the first counter, $N_{2}$ the rate for the second, and $\tau$ is the "resolving power" of the apparatus used to detect coincidences. Putting $N_{1}=N_{2}=30$ per minute and $\tau=10^{-3}$ sec., $E$ becomes 1.7 per hour. For the case of four counters

$$
E=2^{3} \cdot N_{1} N_{2} N_{2} N_{4} \tau^{3}
$$

Putting $N_{1}=N_{2}=N_{3}=N_{4}=30$ per minute and $\tau=10^{-3}$ sec., $E$ drops in this case to 1 coincidence in about 70 years. Consequently it is safe to assume in general that a circuit using four counters only responds to the passage of an ionizing particle through all four counters. A possible exception will be mentioned in the discussion of the results.

The vacuum tube circuit devised by Rossi ${ }^{8}$ was adopted, since it is simpler and easier to operate when extended to four counters than any other arrangement which has been tried. The details of the circuit are shown in Figure 1. The output was connected to a telegraph relay which actuated electrically a dial counter. Switches were arranged to shunt out any three counters by applying a negative voltage to the grids of the three vacuum tubes connected with these counters. In this way the rate of counting of the fourth alone could be observed directly, making possible a frequent check of the rate and sensitivity of each counter.

To eliminate errors arising from changes in temperature each counter was inclosed in a thermally insulated box containing a heating coil and thermostat capable of maintaining the temperature constant to within a few tenths of a degree. The electromagnet and counters were also inclosed so that their temperature could be kept uniform by rapid circulation of the air by means of an electric fan. A small radium preparation inclosed in lead was used to test the sensitivity to penetrating $\gamma$-rays.

The geometrical arrangement of the counters with respect to the poles of the electromagnet is shown in Figure 2, which is drawn to scale. The air gap between the poles, $5 \mathrm{~cm}$ long, determined the useful length of the counters. Observations were made with a magnetic

${ }^{8}$ B. Rossi, Nature, vol. 125, p. 136, 1930. 

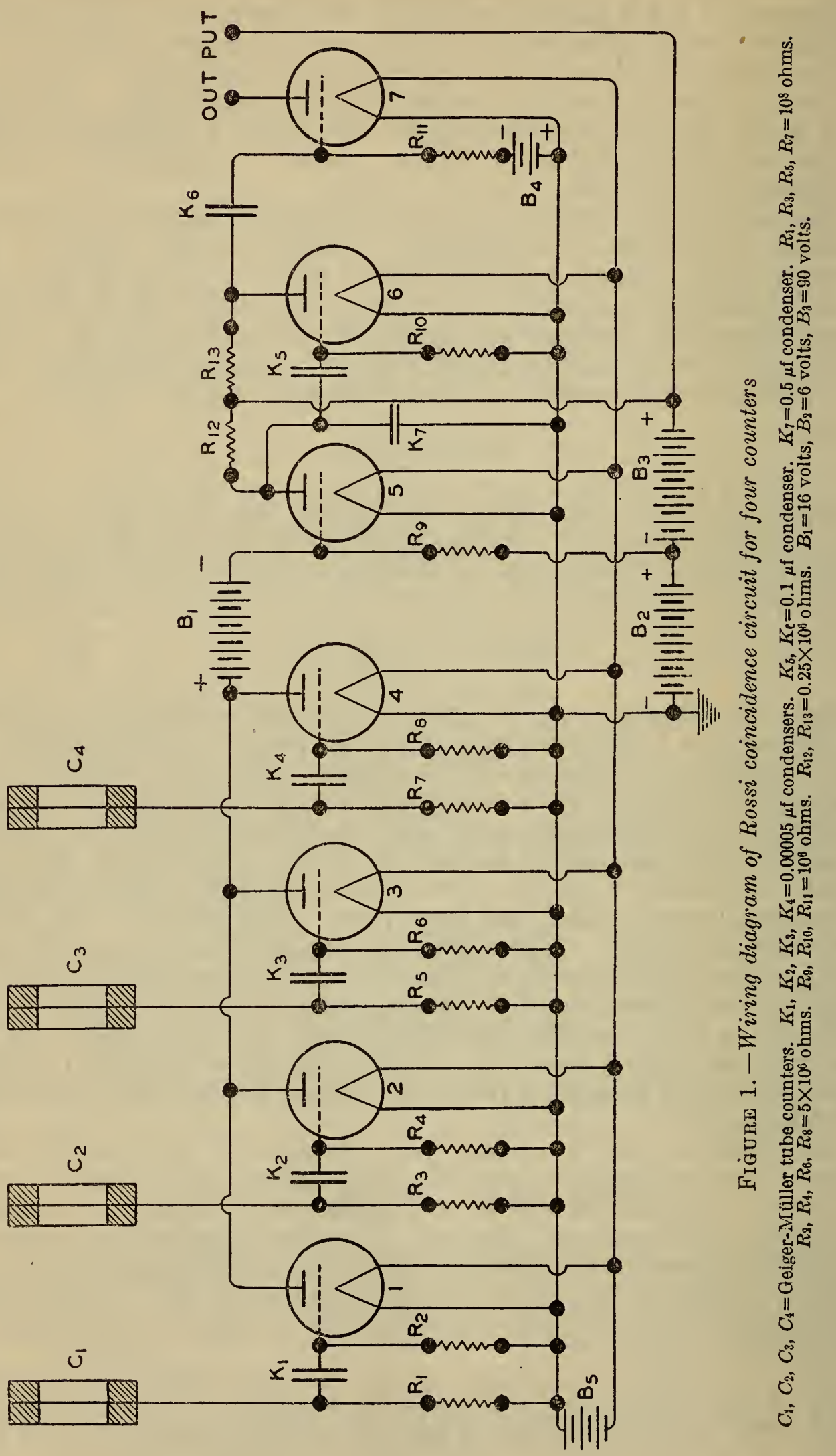
field in the air gap of 7,000 gauss, determined from a calibration curve. As indicated by the dotted lines across the end view of the pole face, a beam of $10^{9}$ volt electrons would, with this arrangement, be deviated just sufficiently to miss the lower counter.

The experiments were carried out under a thin wooden roof with no other filtering, so that the softer components of the cosmic radiation are included in the observations. Since the walls of the counters were of steel, approximately $1 \mathrm{~mm}$ thick, and a particle to produce one coincidence would have to traverse $7 \mathrm{~mm}$ of steel, beta-radiation of radioactive origin could produce very few systematic coincidences.

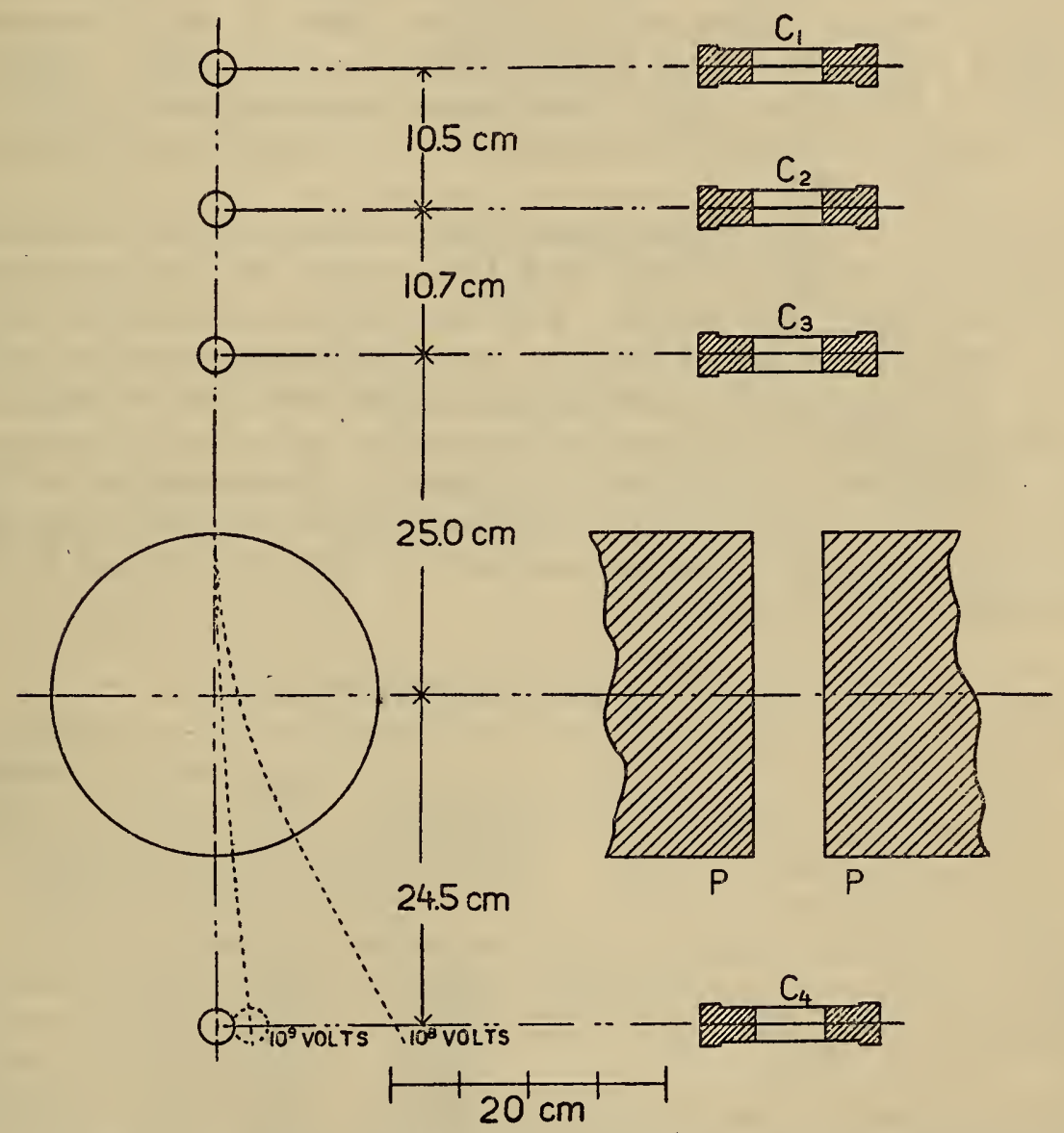

FIGURE 2.-Plan of arrangement of counters with respect to poles of electromagnet

$C_{1}, C_{2}, C_{3}, C_{4}=$ Geiger-Müller tube counters. $P P=$ poles of electromagnet.

Actual observations were preceded by a careful study of the operating characteristics of each counter, making it possible to select for each an operating temperature such that all four could be run at the same voltage. A small 110-volt alternating-current generator driven by a synchronous motor, having its output stepped up by a transformer to about 1,100 volts, supplied this voltage. All parts of this circuit were inclosed and kept at a constant temperature.

\section{OBSERVATIONS}

The experiments were undertaken primarily to determine whether or not cosmic-ray particles could be deflected by a magnetic field. Accordingly, the procedure adopted in taking observations was to 
record the number of coincidences for several hours in the absence of the magnetic field and then to take a similar record with the field applied. This method does not permit a study of the direction of deflections to determine the sign of the charge carried by the particles. The small size of the counters made long periods of observation necessary so that it was decided to settle first, if possible, the question of the existence of a deflection.

A summary of observations is given in the following table. Although in some cases uninterrupted series of observations of a week or more were possible, interruptions frequently occurred. A failure of any part of the complicated arrangements was cause for the rejection of the record taken since the last time it was known to be functioning properly. The chief difficulty arose from temperature variations of the room which was in an unheated building. These variations were sometimes too sudden or too great for the temperature controls to cope with.

As the table shows, observations were made for a total of 636.5 hours with no magnetic field and for a total of 508 hours with a magnetic field of 7,000 gauss. The average number of coincidences without the field was $1.31 \pm 0.09$ per hour and with the field $0.88 \pm 0.14$ per hour. Although, as to be expected under the circumstances, there was a considerable fluctuation in the number of coincidences per hour between individual periods of observation, there can be very little doubt that there were fewer coincidences when the field was applied. The amount of this decrease, as shown by the above data, was approximately 30 per cent.

TABLE 1.-Record of observations

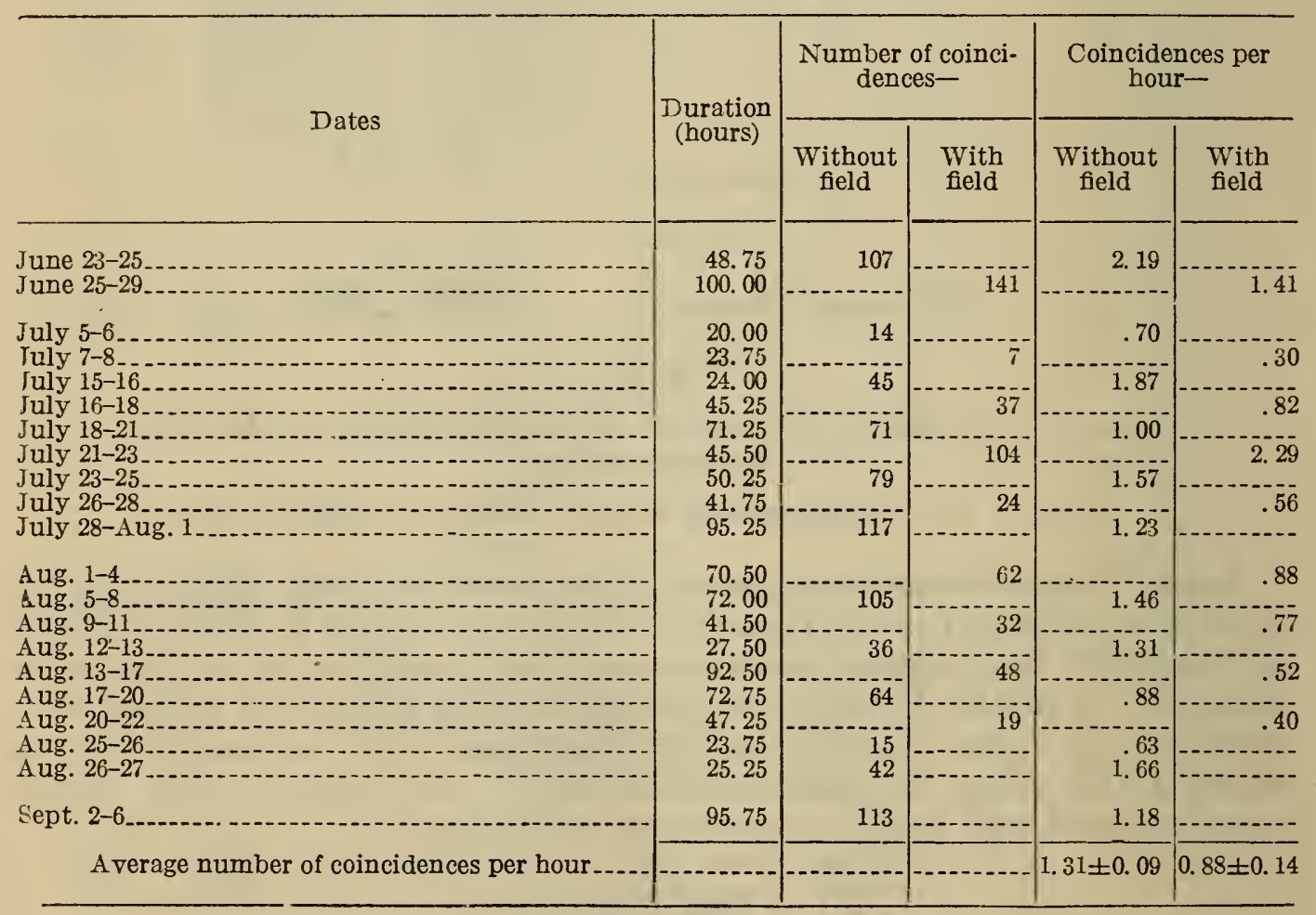




\section{DISCUSSION}

Although the figures given represent only a preliminary approach to the study of the magnetic deviation of cosmic-ray particles, certain conclusions concerning the energy and character of the cosmic-ray particles can be drawn from them. Referring to Figure 2, it is seen that a beam of electrified particles consisting of electrons of energy greater than $10^{9}$ electron volts or protons of energy greater than $5 \times 10^{8}$ electron volts will be detected in the undeflected beam when the magnetic field is applied. This represents 70 per cent of the cosmic rays according to the figures given above. If these undeflected particles have no charge, these experiments reveal nothing concerning their energy. The results could then be explained quite simply by assuming that the primary cosmic-ray beam consists of neutrons. One of the characteristics of the neutron, known at present, is its ability to transmit high velocities to protons and, to a lesser extent, to electrons. Thus, a beam of neutrons penetrating our atmosphere should be accompanied by protons and electrons with high velocities. From the results obtained by Dee ${ }^{9}$ and by Feather, ${ }^{10}$ one would expect the protons to predominate. Thus, if it could be shown that, of the 30 per cent of the beam that shows a deflection, the protons greatly exceed the electrons in number, it could be taken as an indication of the existence of neutrons in the cosmic-ray beam. A more direct attack would be to determine the loss of particles for various strengths of magnetic fields up to the values greatly in excess of that used in the present experiments. It seems reasonable to suppose that under such conditions a point should be found where an increase of strength of the magnetic field produced no further loss of particles. The deviability to be expected for a neutron moving in a magnetic field depends upon the structure assumed for the neutron. As pointed out by Huff, ${ }^{11}$ the model proposed by Pauli may be deflected in a nonhomogeneous magnetic field. However, this deflection is of observable magnitude only when the neutron is traveling at low speeds when it would probably not ionize at all. Consequently, a beam of neutrons, having the high velocities necessary to account for cosmicray phenomena, could not be distinguished by a magnetic experiment from a beam of photons.

In making an attempt to interpret the apparent existence of an undeflected beam of cosmic rays amounting to approximately 70 per cent of the total radiation, the possibility of psuedo coincidences must be considered. Such coincidences are caused by the simultaneous ejection of several secondary particles by a single cosmic ray in such a way that separate secondary particles traverse different counters at the same time. Such an effect has been demonstrated experimentally by Rossi ${ }^{12}$ and by Johnson and Street. ${ }^{13}$ If an appreciable number of the coincidences observed in the present experiments were produced in this way, it would be difficult to arrive at any satisfactory interpretation of the results obtained. It is easy to see in a general way that this could not be true, since the effect is only readily detectable when masses of absorbing material of high atomic number (that is,

\footnotetext{
P. I. Dee, Proc. Roy. Soc., vol. 136, p. 727, 1932.

10 N. Feather, Proc. Roy. Soc., vol. 136, p. 709, 1932.

11 Huff, L. D., Phys. Rev., vol. 38, p. 2292, 1932.

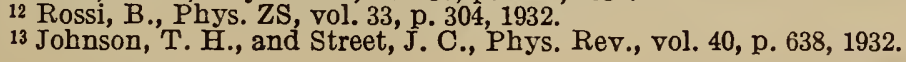
$145879-32-8$ 
lead) are placed slightly above and in the immediate neighborhood of the counters. The secondary particles which cause the pseudo coincidences are projected mainly in the direction of the primary radiation which has its maximum of intensity in the vertical direction. In the present experiments the only large mass of heavy material, the iron of the electromagnet, was below all except one of the counters. 'There was no absorbing material of high atomic number above the counters. Nevertheless it is important to make certain of this by an experimental determination of the probable number of coincidences which might be caused.by this effect. Observations extending over several weeks were made in which one of the counters was placed entirely out of line from the others but near to its original position. The average number of coincidences under these conditions was found to be 0.15 per hour. Although representing about 10 per cent of the total number of coincidences observed with no magnetic field, this figure is but slightly greater than the experimental error. Thus it seems that the effect under discussion presumably did not contribute appreciably to the coincidences observed when all four counters were in line.

The fact that a large proportion of the cosmic rays appear to be less deviable than $10^{9}$-volt electrons seems to offer, when considered in connection with the suggestion made by Johnson, ${ }^{14}$ and explanation for the failure of Rossi, ${ }^{15}$ and of Mott-Smith ${ }^{16}$ to obtain evidence of a deflection when allowing the cosmic rays to pass through magnetized iron. The results given above show that only the softer 30 per cent of the cosmic rays could be expected to show an effect. This is the portion of the beam most easily absorbed or scattered. The scattering of particles in this portion of the beam may be sufficient, when passing through several centimeters of iron, to prevent any of these particles from producing a systematic coincidence.

It is of interest to compare the results obtained here with some of the more recent investigations which offer a basis of comparsion. The remarkable photographs obtained by Anderson ${ }^{17}$ of cosmic ray particles in a Wilson chamber, in the presence of a magnetic field, exhibit particles showing a wide range of magnetic deviations. From the analysis of these photographs which he gives it is seen that of a total of 64 observed particles, 58 have energies over $10 \times 10^{6}$ electron volts and could be expected to produce coincidences through $7 \mathrm{~mm}$ of steel. Only 13 of these 58 particles have energies greater than $400 \times 10^{6}$ electron volts and might, therefore, be considered to form a part of the undeflected beam in the present experiments. These particles are of the energy range where accurate measurements of the Wilson photographs are difficult and, therefore, there may be considerable error in this estimate. On this basis only about 25 per cent of the cosmic-ray beam should have been undeflected in the present experiments as opposed to about 70 per cent observed. Even allowing for the low precision of the observations of coincidences, this discrepancy appears to be genuine, and points to the existence of high-energy cosmic-ray particles of an ionizing power sufficient to actuate a tube counter but difficult to distinguish in a Wilson photograph. This assumption is also supported to some extent by the experiments of

14 Johnson, T. H., Phys. Rev., vol. 40, p. 468, 1932.

16 See footnote 6, p. 816 .

16 See footnote 7, p. 816 .

17 Anderson, C. D., Phys. Rev., vol. 41, p. 405, 1932. 
Johnson, Fleisher, and Street, ${ }^{18}$ in which Wilson photographs were taken only when coincidences occurred between the two tube counters. Although, in the absence of exact information regarding geometrical arrangements and number of possible accidental coincidences, no definite conclusion can be drawn, it is significant that a considerable number of photographs showed no tracks.

\section{ACKNOWLEDGMENTS}

The writer wishes to thank B. W. Brown and L. L. Stockman for help in constructing the apparatus and recording the observations. He is also indebted to Dr. A. V. Astin for helpful discussions of various phases of the experiment.

Washington, October 12, 1932.

18 Johnson, T. H., Fleisher, W., and Street, J. C., Phys. Rev. vol. 40, p. 1048, 1932. 